\title{
Ultrastructural Characterization of Nucleolar Organization in Human Gingival Fibroblast Overexpressing CEMP1
}

\author{
C.E. Villegas-Mercado ${ }^{1}$, L.T. Agredano-Moreno ${ }^{1}$ and L.F. Jiménez-García ${ }^{1}$ \\ 1. Cell Nanobiology Laboratory, Department of Cell Biology. Faculty of Sciences. National Autonomous \\ University of Mexico (Universidad Nacional Autónoma de México-UNAM), Cirucito Exterior, C.U. \\ 04510 Coyoacán, México D.F., México.
}

Periodontal disease affects a large part the population worldwide ${ }^{[1]}$. Currently, numerous treatments have been tested for this condition, nevertheless none is able to achieve the periodontium regeneration ${ }^{[2]}$. The main limitation is the cementum neoformation to restore the insertion of the periodontal ligament. Another problem is the fact that it is difficult to obtain and work with cementoblasts making more difficult the study of cementogenesis ${ }^{[3]}$.

Recently, it was shown that Human Gingival Fibroblasts (HGF) overexpressing CEMP1 (HGF-CEMP1) change its phenotype and present putative cementoblasts characteristics, expressing molecules related to the biomineralization process ${ }^{[4]}$. These changes in cell activity caused by gene transfection may also be accompanied by changes in nucleolar ultrastructure to adapt to the new cell's needs. These changes could be very important since it can impulse the studies related to cementogenesis and periodontal regeneration. Nevertheless, little is known about the ultrastructural changes in the nucleolar organization that take place in the HGF when CEMP1 is overexpressed ${ }^{[5]}$.

Therefore, the aim of this work is to investigate the ultrastructural characterization of Nucleolar Organization of HGF-CEMP1 comparing them with HGF, empty vector HGF (HGF-EV) and cementoblasts (CEM).

To this end, HGF-CEMP1, HGF, HGF-EV and CEM where cultured. Cells between the 2nd and 5th passage were used for the experiments. The cells were grown in DMEM media supplemented with 10\% FBS in a 5\% CO2 and 95\% air atmosphere in a 100\% humidity. The cells were fixed for $1 \mathrm{~h}$ at room temperature in a mixture of $6 \%$ glutaraldehyde and $4 \%$ paraformaldehyde, buffered in PBS (pH 7.2). Postfixation was done in $1 \%$ osmium tetraoxide for $4 \mathrm{~h}$. Samples were subsequently dehydrated in a graded series of ethanol and embedded in an epoxy resin. Semithin sections were stained with toluidine blue. Thin sections of 40-70 nm thickness were contrasted with uranyl acetate and lead citrate.

We found that HGF-CEMP1 cells share similarities in number, form and nucleolar position with CEM cells, indicating that CEMP1 transfection produces a cementoblastic phenotype change in HGF cells [table1]. HGF-CEMP1 presented compact nucleoli while the other groups presented nucleoli with nucleolonema [Figure 1]. This indicates that CEMP1 could induce cells to be more active metabolically. Besides it has been proved that compact nucleoli are associated with a higher ECM secretory activity, which plays an important role in biomineralization processes such as cementogenesis. So we can infer that CEMP1 can also participate in the ECM regulation. The nucleolus may be used as a marker of such processes. [6] 
References:

[1] Kassebaum NJ et al. J Dent Res, 93 (11), 2014, 1045-1053.

[2] Rios HF et al, J Periodontol, 82(9), 2011, 1223-1237.

[3] Arzate H et al, Periodontology 2000, Vol. 65, 2014, 1-24.

[4] Carmona-Rodríguez B et al, Biochem Biophys Res Commun, Jul 6;358(3), 2007, 763-9.

[5] Schwarzacher HG, Wachtler F, Hum Genet, Vol 63, 89-99.

[6] The authors acknowledge funding from Conacyt 180835

\begin{tabular}{|c|c|c|c|c|}
\hline $\begin{array}{c}\text { Nucleolar } \\
\text { Characteristics }\end{array}$ & HGF & HGF-CEMP1 & HGF-EV & CEM \\
\hline 1 & & N u m b e r & & \\
\hline 2 & 57 & 87 & 46 & 72 \\
\hline 3 & 32 & 12 & 37 & 26 \\
\hline 4 & 11 & 1 & 11 & 1 \\
\hline & 0 & 0 & 6 & 1 \\
\hline Round & 65 & S h a p e & & \\
\hline Piriform & 20 & 54 & 58 & 36 \\
\hline Oval & 26 & 19 & 10 & 27 \\
\hline Irregular & 29 & 28 & 38 & 19 \\
\hline Elongated & 14 & 7 & 92 & 30 \\
\hline & & L o c a t i o n & & 19 \\
\hline Central & 54 & 35 & 28 & 35 \\
\hline Near by & 50 & 52 & 87 & 64 \\
\hline In Contact & 50 & 27 & 62 & 32 \\
\hline
\end{tabular}

Table 1. 100 cells of each cell line were analyzed in order to register the nucleolar characteristics as the number of nucleoli per cell, shape and its localization regarding the nuclear envelope. More cells were found with a single nucleolus in all cell lines. Irregularly shaped nucleoli predominate in HGF-EV while in the others cell lines predominate round shape. In position HGF-CEMP1 and CEM have a very similar distribution.
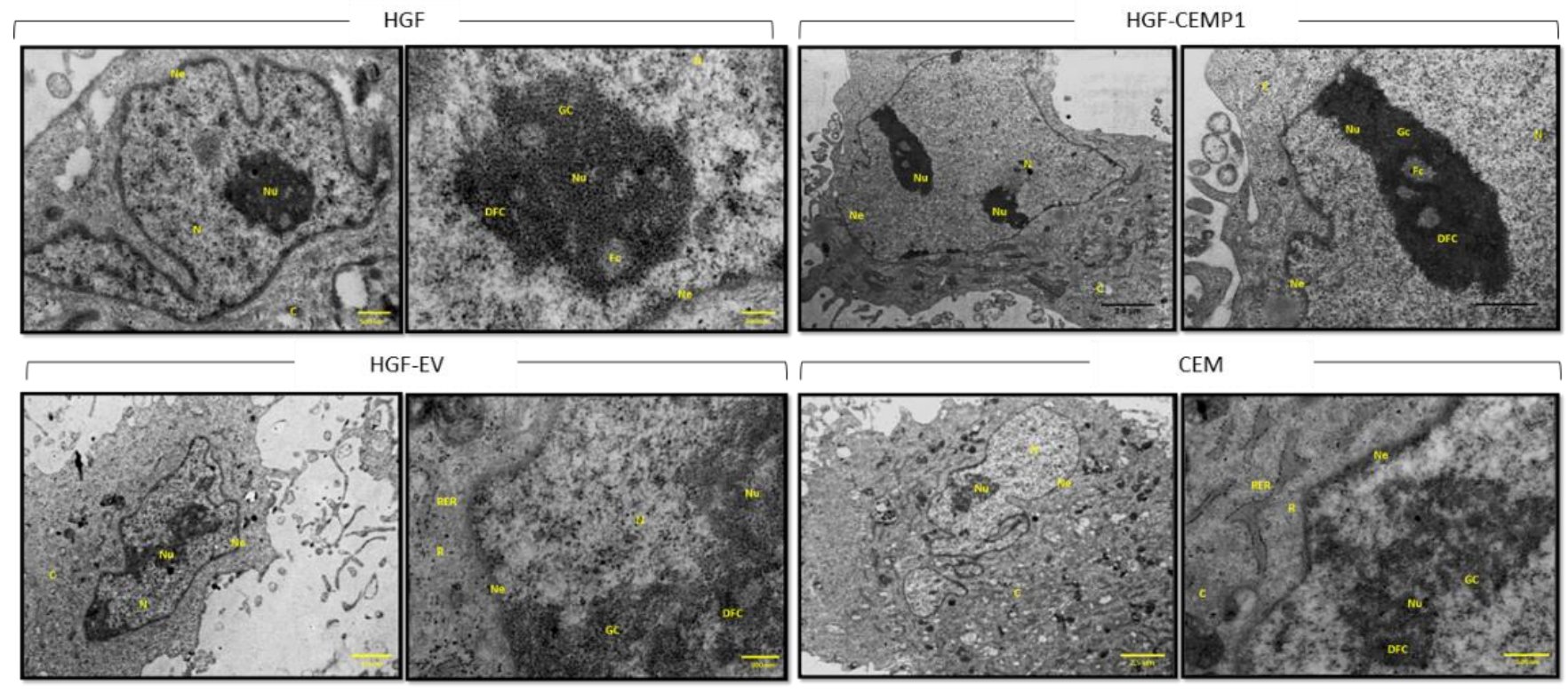

Figure 1. Electron micrographs contrasted with uranyl-acetate-lead citrate. HGF-CEMP1 shown compact nucleolus while the others cell lines present nucleolus with nucleolonema. N, Nucleus; Nu, Nucleolus; Ne, Nuclear Envelope; C, Cytoplasm; RNPs, Ribonucleoproteins; Fc, Fibrillar Center; DFC, Dense Fibrillar Component; GC, Granular Component; CIG, Cluster of Interchromatin Granules; RER, Rough Endoplasmic Reticulum; R, Ribosomes; Cr, Chromatin; 